\title{
Effects of carfilzomib alone and in combination with cisplatin on the cell death in cisplatin-sensitive and cisplatin-resistant ovarian carcinoma cell lines
}

\author{
Zarei $\mathrm{S}^{1}$, Zavar Reza $\mathrm{J}^{1}$, Zarei Jaliani $\mathrm{H}^{1,2}$, Hajizadeh $\mathrm{MR}^{1,3}$, Sargazi $\mathrm{S}^{1,4}$, Hosseinian $\mathrm{H}^{1,5}$ \\ Department of Clinical Biochemistry, School of Medicine, Shahid Sadoughi University of Medical Sciences, \\ Yazd, Iran. Jzavar@ssu.ac.ir
}

\begin{abstract}
BACKGROUND: Previous studies on the efficacy of platinum-based drugs and selective inhibitors of proteasome have revealed promising outcomes. This study is aimed to evaluate the effects of the combination of cisplatin and carfilzomib on the cell death induction and drug efflux transporters expression in cisplatin-sensitive (A2780s) and cisplatin-resistant (A2780cp) ovarian cancer cells lines.

METHODS: MTT cytotoxic assay was conducted to determine the cytotoxicity. Drug interactions were analyzed based on Chou-Talalay's principles and real-time PCR analysis was performed to determine possible alterations in mRNA levels of MRP1 and BCRP.

RESULTS: A2780s cells were more susceptible to both cisplatin and carfilzomib while analyses of drug interactions between the two agents showed synergistic effects in all affected fractions of drug-treated A2780s and A2780cp cells $(\mathrm{Cl}<0.9)$ with the combination indices being significantly lower in A2780cp cells $(p<0.01)$. We also found that although mRNA levels of BCRP and MRP1 were significantly altered in both cells exposed to each drug alone, only the combination regimen was able to significantly reduce the mRNA levels of these genes in A2780cp cells $(p<0.001)$.

CONCLUSION: This combination might be a potential strategy for suppressing cell growth via downregulating the drug efflux transporters expression, especially in cisplatin-resistant ovarian cancer cells (Fig. 3, Ref. 45). Text in PDF www.elis.sk.

KEY WORDS: cisplatin, carfilzomib, drug combination, ovarian neoplasms.
\end{abstract}

\section{Introduction}

As reported by many researches, the highest mortality rates among female genital cancers belong to ovarian cancer (OC) which are also the seventh most common cancer (1). Since 2012, 239000 women have been diagnosed with OC worldwide where 152000 of them did not survive. In Iran, OC has been identified as the eighth leading cause of death in 2008 (2). OC is a heterozygous

\footnotetext{
${ }^{1}$ Department of Clinical Biochemistry, School of Medicine, Shahid Sadoughi University of Medical Sciences, Yazd, Iran, ${ }^{2}$ Protein Engineering Laboratory, Department of Medical Genetics, School of Medicine, Shahid Sadoughi University of Medical Sciences, Yazd, Iran, ${ }^{3}$ Molecular Medicine Research Center, Dept. of Biochemistry, Rafsanjan University of Medical Sciences, Rafsanjan, Iran, ${ }^{4}$ Cellular and Molecular Research Center, Zahedan University of Medical Sciences, Zahedan, Iran, and ${ }^{5} \mathrm{De}-$ partment of Biology, Faculty of Science and Engineering, Science and Arts University, Yazd, Iran

Address for correspondence: J. Zavar Reza, Dr, Department of Clinical Biochemistry, School of Medicine, Shahid Sadoughi University of Medical Sciences, Yazd, Iran.

Phone/Fax: +983538202633
}

Acknowledgments: This work in financially supported by Shahid Sadoughi University of Medical Sciences, Yazd, Iran. disease in histopathology and molecular biology, which can spread to other tissues such as abdominal wall, lymph nodes, lungs and liver tissues $(3,4)$. The risk of developing OC is higher in those who are multiply ovulated compared with those who have never been pregnant. Also, the use of fertility drugs, obesity and initiation of ovulation at an earlier age or at a higher post-menopausal age are also considered risk factors of $\operatorname{OC}(5,6)$. To this date, no definitive treatment for $\mathrm{OC}$ has been found, mostly due to the complexity of the mechanisms and the presence of several factors in the development of the disease. Therefore, in recent years, new strategies have been introduced to prevent and treat patients diagnosed with OC (7).

Cisplatin, one of the most efficacious alkylated agents, can be used alone or in combination with other chemotherapy drugs to treat breast, ovarian, lung, and other solid tumors. This platinumbased drug mostly binds to the heterocyclic purine bases of DNA. Accumulation of damaged DNA in the cancer cells eventually leads to blocking the cell division, and cell death (8). Nevertheless, in spite of the initial efficacy of cisplatin, long-term use can cause drug resistance that has remained a major barrier to the efficacy of chemotherapy in OC patients (9). Increased expression of the drug transporters and enhanced activation of glutathione $\mathrm{S}$ transferase appear to be the two main causes of inducing 
drug resistance (10). Among those, up-regulation of multidrug resistance protein 1 (MRP1) and breast cancer resistance protein (BCRP) have been established to have a significant impact on gaining drug resistance in OC cells, including cisplatin-resistance A2780 (A2780cp) cell line (11), whereas cisplatin-sensitive A2780 (A2780s) cells display considerably lower levels of drug efflux transporters. On the other hand, increased levels of proteasome-ubiquitin stress have been previously observed in OC cells in comparison with normal cells (12) which probably has led to the hypothesis that genetically unstable malignant cells are often more susceptible to inhibitors of the proteasome activity than healthy tissue (13). Large proteolytic complexes of proteasome are normally labeled with Ubiquitin which systematically destroys more than $80 \%$ of regulatory proteins associated with regulation of cell cycle, cell death and differentiation by spontaneously increasing the accumulation of pro-apoptotic proteins and decreasing the activity and degradation of NF-kB $(14,15)$. Studies have revealed that selective proteasome inhibitors induce cell death by making them susceptible to chemotherapeutic agents in the breast (16), prostate (17), pancreatic (18), and ovarian cancers (19). In 2012, Carfilzomib (an epoxomycin derivative) has been approved by the Food and Drug Administration (FDA) as the second generation proteasome inhibitor for the treatment of multiple myeloma, showing lower toxicity than the classic proteasome inhibitors (20). Carfilzomib irreversibly binds to the catalytic site of the proteasome chymotrypsin $20 \mathrm{~S}$ and induces a cellular stress response and therefore initiates apoptosis (13). It has been estimated that up to $60 \%$ of patients treated with bortezomib (the first generation of proteasome inhibitors) have shown side effects or median resistibility through one year of treatment (21). Unlike bortezomib, carfilzomib can be given in successive days $(22,23)$. Carfilzomib suppresses the cell cycle in the G2/M phase and induces apoptosis $(24,25)$.

Several studies have argued the promising outcomes of combining proteasome inhibitors with conventional drugs, expecting to inhibit tumor growth at higher rates rather than monotherapeutic strategies $(26,27)$. In this study, we examined the cytotoxic effects and the possible changes in mRNA levels of two drug efflux transporters in A2780s and A2780cp ovarian cancer cell lines treated with carfilzomib, cisplatin, and their combination.

\section{Material and methods}

The protocol of this study has been approved by the Ethics Committee of Shahid Sadoughi University of Medical and Health Services, Yazd, Iran (IR.SSU.MEDICINE.REC.1396.53).

\section{Drug, chemicals and cell culture conditions}

Carfilzomib and cisplatin were purchased from AdooQ BioScience (CA, USA) and dissolved in dimethyl sulfoxide (DMSO) (Pars Tous Biotechnology, Mashhad, Iran) and stored at -20 ${ }^{\circ} \mathrm{C}$ until further use. Cisplatin was purchased from Sigma-Aldrich (St. Louis, MO, USA) and freshly solubilized in predetermined amounts of phosphate buffer with a relatively acidic $\mathrm{pH}$, and kept away from light. RNA extraction kit was obtained from
RiboEx (GeneAll, Korea) and SYBR Green master mix and cDNA synthesis kits were procured from Ampliqon A/S (Odense M, Denmark) and CinaColon (Tehran, Iran), respectively. 3-(4,5- dimethylhiazol- 2-yl)-2,5-diphenyltetrazolium bromide (MTT), Trypan blue and Trypsin-EDTA were all purchased from from INOCLON (G. Innovative Biotech Co (INOCLON), Iran).

The A2780s and A2780cp ovarian cancer cells were procured from Pasteur Institute of Iran (Tehran, Iran) and incubated in RPMI1640 (Gibco, Thermofisher, USA) supplemented with 10 $\%$ inactivated fetal Bovine serum (Gibco, Thermofisher, USA), $100 \mathrm{U} / \mathrm{mL}$ penicillin (Sigma-Aldrich, St. Louis, MO), $100 \mathrm{U} / \mathrm{mL}$ streptomycin (Sigma-Aldrich, St. Louis, MO) at $37{ }^{\circ} \mathrm{C}$ in $5 \%$ $\mathrm{CO}_{2}$ and $95 \%$ humidity. The A2780cp cell line was treated with cisplatin $(1 \mu \mathrm{M})$ up to three passages before conducting the following assays. Cells treated with DMSO (up to $2 \%$ ) were considered as control group.

\section{Cell viability assay and analysis of drug interactions}

The half-maximal inhibitory concentration (IC50) of carfilzomib and cisplatin for both cell lines was assessed by the MTT cytotoxic assay (28). A2780s and A2780cp cells at 10,000 cells/ well were seeded into 96 well plates and incubated in normal cell culture conditions to reach $80 \%$ of confluency. Then, both cells were treated by a wide range of carfilzomib $\left(10-512 \times 10^{-8} \mu \mathrm{g} / \mathrm{mL}\right)$. A2780s and A2780cp cells were also exposed to 0.005 to $100 \mu \mathrm{g} /$ $\mathrm{mL}$, and 0.025 to $500 \mu \mathrm{g} / \mathrm{mL}$ of cisplatin, respectively, following 24, 48, and 72 hours. Afterward, $0.5 \mathrm{mg} / \mathrm{mL}$ of MTT dye was added to each well and following 4 hours of incubation at $37^{\circ} \mathrm{C}$, $100 \mu \mathrm{L}$ DMSO was added and the absorbance was measured at $570 \mathrm{~nm}$ according to the following equation;

Cell viability $=$ absorption of treated cells $[570 \mathrm{~nm}] /$ absorption of untreated cells $[570 \mathrm{~nm}] \times 100$.

Then both cell lines were treated with both drugs (as a single agents) at the following concentration ranges: Cisplatin: 500-0.025 $\mu \mathrm{g} / \mathrm{mL}$; carfilzomib: $10-512 \times 10^{-8} \mu \mathrm{g} / \mathrm{mL}$ and cisplatin: $100-0.005$ $\mu \mathrm{g} / \mathrm{mL}$; carfilzomib: $10-512 \times 10^{-8} \mu \mathrm{g} / \mathrm{mL}$ with combination ratio of 1:50 and 1:10 for A2780cp and A2780s cells, respectively. For analysis of drug combination based on Chou-Talalay method and median-effect principles, CompuSyn software (Version 1.0, Combo-Syn Inc., US) was applied to determine the mode of interaction between cisplatin and carfilzomib combination based on combination indices (CIs) where $\mathrm{CI}>1.1, \mathrm{CI}=0.9-1.1$ and $\mathrm{CI}<0.9$ simply indicates antagonism, additive mode, and synergism, respectively (29).

\section{Total RNA extraction and cDNA synthesis}

Using protocol for RNA extraction, total RNA was extracted after 24,48 , and 72 hours of exposure to both drugs alone and in combination with concentrations equal to IC50 values of the corresponding treatment period. RNA quality and integrity were checked using a NanoDrop (Biotek) and gel electrophoresis. After thawing, the components of the kit were mixed, briefly centrifuged, and stored on ice. RNA-primer mixture prepared in 

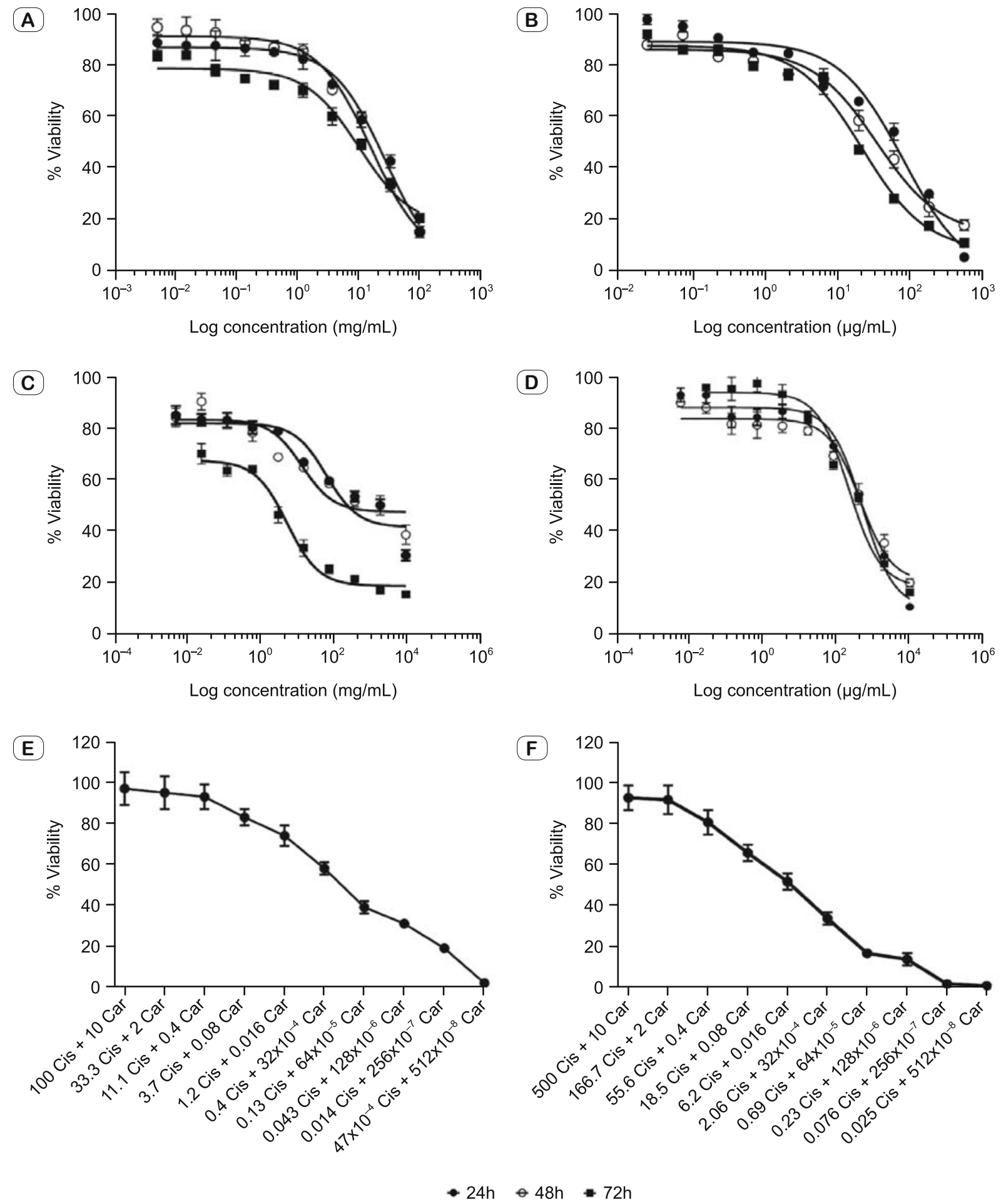

Fig. 1. Dose-response curves associated with the treatment of A2780cp cells with cisplatin (A) and carfilzomib (B) and A2780s cells with cisplatin (C) and carfilzomib (D) for 24, 48, 72 hours. Following 72 hours of co-treatment with both agents (in terms of $\mu \mathrm{g} / \mathrm{mL}$ ), section (E) and (F) shows the dose-response curves for A2780s and $\mathbf{A 2 7 8 0 c p}$, respectively. Each value is presented as a mean \pm SD of three independent experiments. Cis $=$ cisplatin and Car $=$ carfilzomib. 
a sterile, nuclease-free tube on ice and incubated at $65^{\circ} \mathrm{C}$ for 5 minutes and instantly chilled on ice for 2 minutes. Then, the mixture was gently spun down and cDNA synthesis mix was prepared. $10 \mu \mathrm{L}$ of the cDNA Synthesis Mix was added into each RNA-primer mixture and immediately incubated at $42{ }^{\circ} \mathrm{C}$ for $60 \mathrm{~min}$. Then, $0.5 \mu \mathrm{L}$ random hexamer and $0.5 \mu 1$ oligo (dt) were subsequently added to the mixture and incubated at $42^{\circ} \mathrm{C}$ to prevent dissociation of primer from the template while the temperature increases. The reaction was terminated by incubation at $85^{\circ} \mathrm{C}$ for $5 \mathrm{~min}$. Finally, the synthesized cDNA was stored at $-20^{\circ} \mathrm{C}$.

\section{Real-time PCR (qPCR)}

In order to perform realtime-PCR, primers of MRP1 (NCBI Reference Sequence: NG_028268.1), BCRP (NCBI Reference Sequence: NG_017013.2), and GAPDH (NCBI Reference Sequence: NG_007073.2) genes were designed by use of the AlleleID software. The MRP1 primers were 5'-TGTTGGTTGCTTACAGTGTTG-3' (forward) and 5'-TGGGAGGTATTTCGTGTTCTT-3' (reverse), the $P 53$ primers were 5'-GCTCAGATAGCGATGGTCTGG-3' (forward) and 5'-CTGTCATCCAAATACTCCACACG-3' (reverse), and the GAPDH primers as reference gene were 5'-CTCATTTCCTGGTATGACAACGA-3' (forward) and 5'-TCTTCCTCTTGTGCTCTTGCTG-3' (reverse). The amplification conditions were: 3 minutes $/ 95^{\circ} \mathrm{C}, 40$ cycles each consisted of denaturation for 35 seconds at $95{ }^{\circ} \mathrm{C}$; annealing for 30 seconds at $55^{\circ} \mathrm{C}$ (for $M R P 1$ ) and $52^{\circ} \mathrm{C}$ (for $B C R P$ ) followed by an extension for 45 seconds at $72{ }^{\circ} \mathrm{C}$. Using the comparative $2^{-\Delta \Delta \mathrm{Ct}}$ method (30), fold changes in mRNA levels of both genes were determined.

\section{Statistical analysis}

Data were analyzed using SPSS (version 16, SPSS Inc., Chicago, IL) and presented as mean \pm standard deviation (SD). When appropriate, non-parametric tests such as One-way ANOVA and sample T-test were applied to compare different groups where $\mathrm{p}<$ 0.05 was regarded statistically significant. The inhibition assay data was modeled with a nonlinear regression fit of the Log[inhibitor] vs. normalized response (using a variable slope) in GraphPad Prism software. All experiments were repeated three times.

\section{Results}

\section{In vitro cytotoxicity and analysis of combination}

Figures 1A, B demonstrates the dose-response curves when A2780cp cells were exposed to mentioned concentrations of cisplatin and carfilzomib whereas Figure $2 \mathrm{~B}$ and $\mathrm{C}$ represent the dose-response curve of A2780s cells treated with different concentrations of cisplatin and carfilzomib at the same exposure periods, respectively. The IC50 values for exposing A2780cp cells to cisplatin were $27.72,16.16$ and $10.37 \mathrm{mg} / \mathrm{mL}$ after 24,48 and 72 hours of treatment, respectively, while these values were $0.067,0.014$ and $0.006 \mathrm{mg} / \mathrm{mL}$ for $\mathrm{A} 2780 \mathrm{~s}$ cells in the same treatment periods, indicate that this alkylating agent can reduce the viability of both cell lines in a dose-dependent mode. Regarding the treatment of both cells with carfilzomib, the IC50 values following 24 hours
( 75.08 and $0.570 \mu \mathrm{g} / \mathrm{mL}), 48$ hours $(32.25$ and $0.436 \mu \mathrm{g} / \mathrm{mL}$ ), and 72 hours $(20.55$ and $0.218 \mu \mathrm{g} / \mathrm{mL}$ ) for A2780cp and A2780s cells, respectively, suggest that this novel proteasome inhibitor is able of causing significant cell-death following a dose- and time-dependent fashion as well with much higher IC50 values in A2780cp cells compared with that of A2780s cells. Following 72 hours of treatment, the dose-response curves of cisplatin+carfilzomib combinations with mentioned ratios are depicted in Figures 1E and F. Combination analysis performed by compuSyn software revealed that combination of carfilzomib and cisplatin mediates synergistic effects in both cell lines $(\mathrm{CI}<0.9)$ effective doses being significantly lower in A2780cp cells compared with A2780s cell line (Fig. 2).

\section{Effects of cisplatin, carfilzomib and their combination on MRP1 and BCRP mRNA levels}

As shown in Figure 3AA2780cp cells displayed significantly higher BCRP and MRP1 mRNA levels compared to A2780s cells $(p<0.01)$. Cisplatin significantly down-regulated BCRP and $M R P 1$ expressions of A2780s cells while this drug caused significant increases in mRNA levels of both genes following 48 and 72 hours of treatment (at 125 , and $110 \%$ of the control after 48 hours and 147 , and $120 \%$ of the control after 72 hours) in A2780cp, respectively $(p<0.01)$. Additionally, carfilzomib was observed to be able to decrease the BCRP after 48 hours ( $93 \%$ of the control in A2780cp cells and $54 \%$ in A2780s cells) and 72 hours (63\% of the control in A2780cp cells and $41 \%$ in A2780s cells), while these values were $83,39,53$ and $28 \%$ for MRP1, showing that this proteasome inhibitor down-regulates the expression of drug efflux proteins in both cells but more significantly in cisplatinsensitive A2780s cells. The real-time-PCR analysis of the combination of both drugs disclosed that co-treatment of A2780s cells with cisplatin+carfilzomib markedly decreased BCRP and MRP1 mRNA levels compared with that of either agent alone following 48 and 72 hours in both cells, while this down-regulation was more significant in A2780s cells exposed to both drugs for 72 hours (10 and $16 \%$ of the control for BCRP and 7 and $12 \%$ of the control for MRP1) (Fig. 3B, C) $(\mathrm{p}<0.001)$.

\section{Discussion}

Although various studies have established that the use alkylating agents as chemotherapeutic therapy substantially improved overall survival compared to single-agent therapy in vitro and in vivo $(31,32)$, the prognosis of advanced ovarian cancer remains poor, and novel treatments demonstrating favorable toxicity profiles are urgently required. Therefore, we aimed to combine a classic anti-tumor agent with a recently developed proteasome inhibitor as a well-designed molecular strategy to overcome drug resistance in such solid tumors. As far as we know, this is the first study focused on determining the drug interactions between cisplatin and carfilzomib and evaluation of possible changes in mRNA levels of MRP1 and BCRP in ovarian cancer cell lines displaying different sensitivity to cisplatin. Maria Florea et al declared that cisplatin is able to induce cytotoxicity by various mechanisms, 

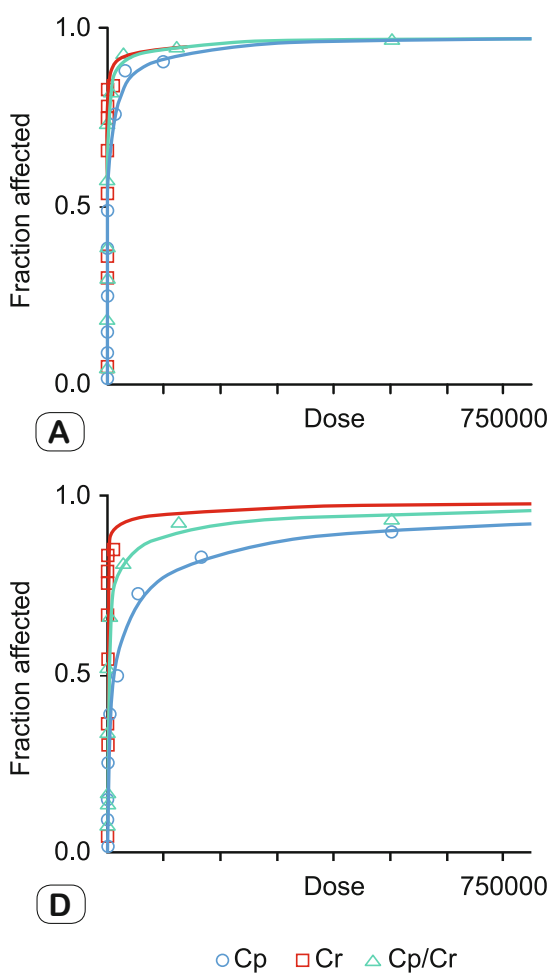

G

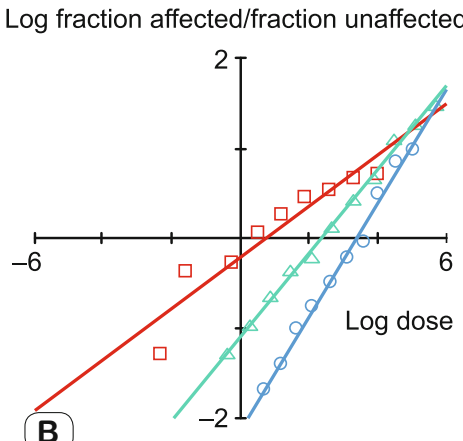

Log fraction affected/fraction unaffected

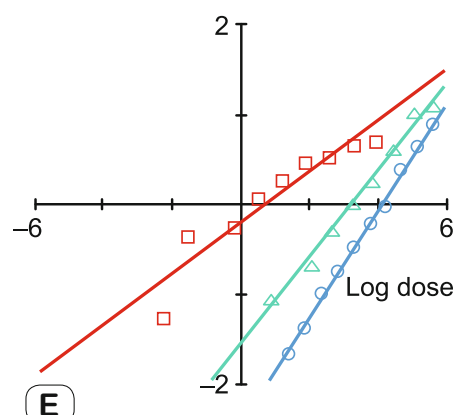

(E)

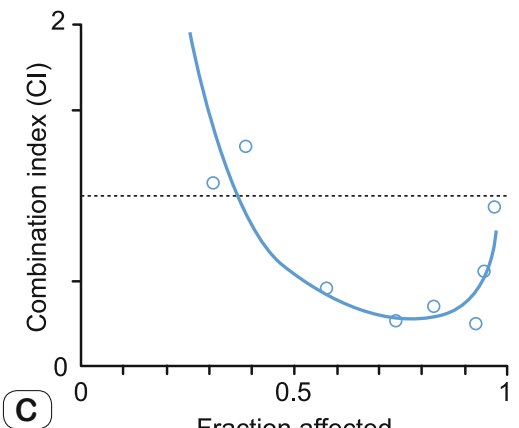

Fraction affected

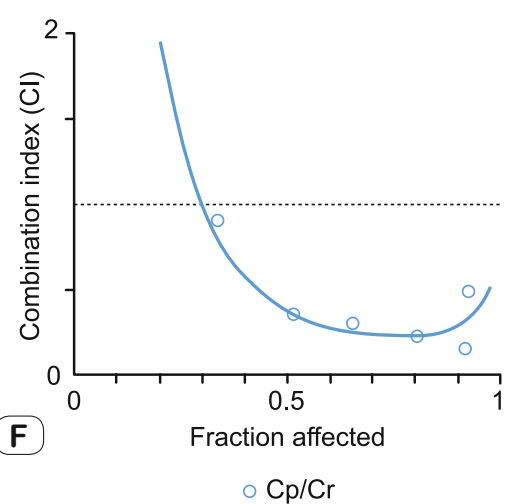

A2780S

A2780Cp

Fig. 2. Cisplatin and carfilzomib combination analysis in cisplatin-sensitive and -resistant $\mathrm{OC}$ cells following 72 hours treatment depicted by Compusyn software. Dose-effect curve (A), median-effect plot (B), and combination index plot (C) represent the efficacy of this regimen in A2780s cells while Dose-effect curve, median-effect plot, and combination index plot for A2780cp cells are shown in section (D), (E) and (F), respectively. CI values at various effective doses (EDs) (G) of drug combination are significantly lower in A2780cp cells $(* * p<0.01 \mathrm{compared}$ to $\mathbf{A 2 7 8 0 s}$ cells).

e.g., by interference with DNA replication and/or transcription (9). Accordingly, this alkylating agent has shown its potential as an apoptosis inducing chemical by activating intrinsic and extrinsic apoptotic pathways beside initiating calcium signaling pathway (33). Regrettably, neither anti-proliferative nor cell-inducing potential of cisplatin are solely induced in ovarian tumor cells. Thus, this drug might cause unpleasant sideeffects including renal- and/or neurotoxicity. In this scenario, a combination-chemotherapy with cisplatin is considered as a cornerstone for the treatment of various solid tumors including advanced ovarian cancer, but the challenge is that cisplatinbased adjuvant chemotherapy could lead to cisplatin-resistance in patients. The principal mechanisms underlying cisplatin resistance have been well distinguished as we focused on the in- hibition of apoptosis and modulating drug efflux in the current study. As well as cisplatin, carfilzomib as a highly cytotoxic proteasome inhibitor basically tends to target the chymotrypsinlike (CT-L) subunits in both the immunoproteasome (i20S)- and constitutive proteasome (c20S). Accordingly, Parlati et al announced that selective inhibition of each of the two proteasome subunits was proved to be cytotoxic for hematologic tumor cells (34). Also, antitumor activity of carfilzomib was observed at doses of $\geq 11 \mathrm{mg} / \mathrm{m}^{2}$ in a study conducted by O'Connor et al, highlighting the clinical activity and tolerability of this proteasome inhibitor in multiple hematologic malignancies (35). Despite the multitude of reported investigations concerning the evaluation of anti-proliferative effects of carfilzomib in patients diagnosed with hematological disorders, few studies were conducted in order to 
A

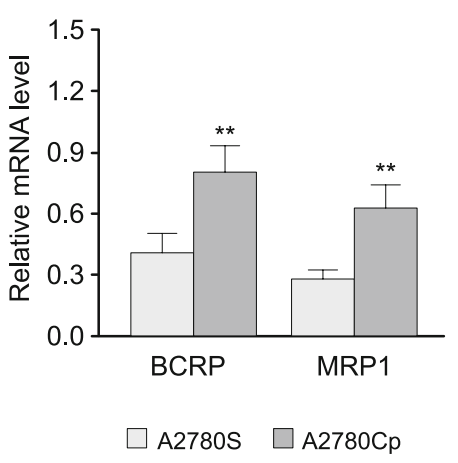

B

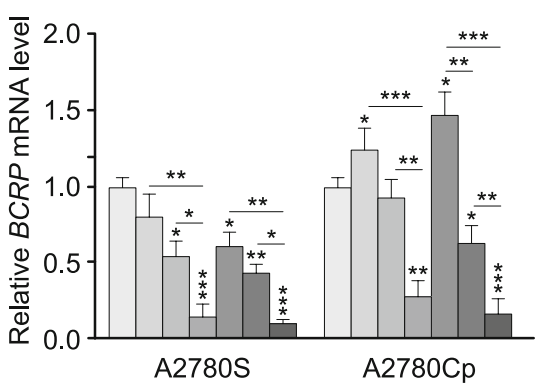

(C)

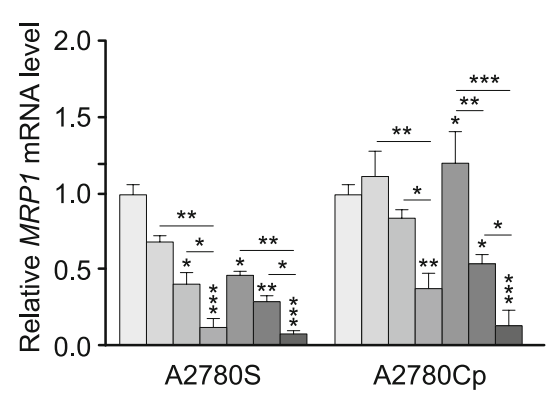

$\square 0 \mathrm{~h} \quad \square 48 \mathrm{~h}$ Cis $\square 48 \mathrm{~h}$ Car $\square 48 \mathrm{~h}$ Cis + Car $\quad \square 72 \mathrm{~h}$ Cis $\quad \square 72 \mathrm{~h}$ Car $\square 72 \mathrm{~h}$ Cis + Car

Fig. 3. Real-time PCR analysis of BCRP (A) and MRP1 (B) mRNA expression in 24, 48, and 72 hours after co-treatment of both cell lines with single drugs and their combination with concentrations equal to their corresponded IC50 values $(* \mathbf{p}<0.05, * * \mathbf{p}<0.01$, and $* * * p<0.001$ compared to untreated adjusted cells).

assess the cytotoxic effects of carfilzomib on ovarian cancer cells as well as no proof of considering this drug as a single strategy to overcome drug resistance in this type of malignancies. In our study, MTT assay results indicated that A2780cp cells showed resistance to both cisplatin and carfilzomib with 24 to 96 hours treatment (with higher IC50 values), founding the hypothesis that OC cells with relative resistance to cisplatin might be also sensitive to higher concentrations of carfilzomib. As stated in previous studies, the IC50 values of cisplatin were 3 and $30 \mu \mathrm{g} / \mathrm{mL}$ on A2780s and A2780cp cells (36), respectively, while carfilzomib was found to suppress cell-growth of different cancer cell lines by $1 \mathrm{nM}(0.0007 \mu \mathrm{g} / \mathrm{ml})$ to $203 \mathrm{nM}(0.146 \mu \mathrm{g} / \mathrm{ml})(37,38)$ which are not very different compared to our findings. Also, synergistic relationship between cisplatin and carfilzomib was observed in both cisplatin-sensitive and -resistant A2780 cells $(\mathrm{CI}<0.9)$ which was in agreement with findings of Konak et al regarding synergistic effects of bortezomib and cisplatin on human bladder cancer cells by increasing caspase 3 activation (39). In a study conducted by Fribley et al, it was established that PS-341, a proteasome inhibitor, may be utilized for overcoming cisplatin-resistance in human squamous cell carcinoma cells (40). Although both cell lines displayed basal expression levels of drug efflux transporters, $M R P 1$ and $B C R P$ expression was found to be higher in cisplatin-resistant A2780cp cells. Particularly, overexpression of these two genes rendered cells resistant to cisplatin which was previously reported byShen et al (41). Li et al evaluated the possible alterations on mRNA levels of multidrug resistance protein 1 (MRP1) and breast cancer resistance protein $(B C R P)$ as prognostic factors in patients treated with cisplatin and reported that high expression of $M R P 1$ was correlated with shorter tumor-free survival while low $M R P 1$ expression was independent predictor of overall survival of patients struggling with lung cancer (42). Our data indicate that co-treatment of carfilzomib and cisplatin led to a robust downregulation of $M R P 1$ and $B C R P$ compared with that of either agent alone after 48 and 72 hours of treatment. This observation was not only applied to A2780cp cells, validating the previously described synergistic interaction between cisplatin and carfilzomib. As expected, we observed significant changes in BCRP and MRP1 expression compared to cells exposed to each drug alone in both cell lines, but surprisingly this down-regulation was more significant in A2780s cells with lower CI values with respect to the analysis of drug combinations. We also observed that proteasome inhibitors are capable of surpassing drug-mediated efflux of tumor cells that was not different from previously published experiment by Farabegoli et al, introducing Epigallocatechin-3-gallate (EGCG) as an effective proteasome inhibitor which down-regulates the activity of BCRP and MDR1 as two major modulators of drug efflux and transport in tamoxifen-resistant breast tumor cells (43).

Our findings preliminarily showed that deregulation of $B C R P$ and MRP1 is a major recurrent event in OC and could play an essential function in drug resistance. No study has yet reported the combination effects of carfilzomib and alkylating agents in vitro. However, growth suppressing potentials of carfilzomib is well studied in combination with histone deacetylase inhibitors, as Dasmahapatra et al reported that vorinostat potentiates the anti-tumor activity of carfilzomib, both in vitro and in vivo (44). Also, Berdeja et al discovered that the combination of carfilzomib and panobinostat is effective in patients with relapsed/refractory multiple myeloma (45). The outcome of the mentioned studies is completely in agreement with our findings revealing the synergistic effects of carfilzomib+cisplatin in both cisplatin-sensitive and resistant ovarian cancer cell lines. Using apoptosis detection techniques to determine the type of cell-death involved in response to this combination regimen and evaluating the protein levels of drug efflux transporters might need to be further investigated in order to validate our findings.

\section{Conclusion}

Inhibition of apoptosis and drug efflux are among well-known mechanisms of cisplatin resistance causing A2780cp ovarian tumor cells to respond inadequately to platinum-based therapies 
as mono-therapeutic agents. Our results showed that carfilzomib enhances cisplatin sensitivity in OC cells via modulation of the expression of drug efflux transporters. Combination therapies were developed in order to minimize cisplatin resistance in ovarian cancer cells. By understanding the other potential mechanisms triggered by cisplatin alone and in combination with other targeted agents, with designing more efficient therapeutic strategies would be possible to reduce the current side effects of platinumbased drugs.

\section{References}

1. Liu JZ, Milner JT. Age, dietary selenium and quantity of 7, 12-dimethylbenz (a) anthracene influence the in vivo occurrence of rat mammary DNA adducts. J Nutr 1992; 122 (7): 1361-1368.

2. Akbari M, Khayamzadeh. Incidence, mortality and burden of cancers in Iran. Shahid Beheshti University of Medical Sciences Press, 2008: 95-125.

3. Ruddon RW. Cancer biology: Oxford University Press, 2007: 234-245.

4. Feuerstein MJ. Defining cancer survivorship. J Cancer Surviv 2007; 1 (1): 5-7.

5. Stewart B, Wild CP. World cancer report. Lyon: IARC press, 2014 2018. $182-185$

6. Cannistra SA. Cancer of the ovary. New Eng J Med 2004; 351 (24): 2519-2529.

7. Wagner V, Dullaart A, Bock AK, Zweck AJ. The emerging nanomedicine landscape. Nat Biotechnol 2006; 24 (10): 1211-1217.

8. Swift L, Golsteyn R. Genotoxic anti-cancer agents and their relationship to DNA damage, mitosis, and checkpoint adaptation in proliferating cancer cells. Int J Mol Sci 2014; 15 (3): 3403-3431.

9. Florea AM, Büsselberg D. Cisplatin as an anti-tumor drug: cellular mechanisms of activity, drug resistance and induced side effects. Cancers 2011; 3 (1): 1351-1371.

10. Stavrovskaya A. Cellular mechanisms of multidrug resistance of tumor cells. Biochemistry c/c of Biokhimiia 2000; 65 (1): 95-106.

11. Wu W, Liu Y, Ye H, Li Z. Millepachine showed novel antitumor effects in cisplatin-resistant human ovarian cancer through inhibiting drug efflux function of ATP-binding cassette transporters. Phytother Res 2018; 32 (12): 2428-2435.

12. Bazzaro M, Lin Z, Santillan A et al. Ubiquitin proteasome system stress underlies synergistic killing of ovarian cancer cells by bortezomib and a novel HDAC6 inhibitor. Clin Cancer Res 2008; 14 (22): 7340-7347.

13. Parlati F, Lee SJ, Aujay M et al. Carfilzomib can induce tumor cell death through selective inhibition of the chymotrypsin-like activity of the proteasome. Blood 2009; 114 (16): 3439-3447.

14. Suzuki Y, Nakabayashi Y, Takahashi R. Ubiquitin-protein ligase activity of X-linked inhibitor of apoptosis protein promotes proteasomal degradation of caspase-3 and enhances its anti-apoptotic effect in Fas-induced cell death. Proc Natl Acad Sci USA 2001; 98 (15): $8662-8667$.

15. Kao C, Chao A, Tsai $\mathbf{C}$ et al. Phosphorylation of signal transducer and activator of transcription 1 reduces bortezomib-mediated apoptosis in cancer cells. Cell Death Dis 2013; 4 (2): e512.
16. Wang H, Yu Y, Jiang $Z$ et al. Next-generation proteasome inhibitor MLN9708 sensitizes breast cancer cells to doxorubicin-induced apoptosis. Sci Rep 2016; 6: 26456.

17. Aras B, Yerlikaya AJ. Bortezomib and etoposide combinations exert synergistic effects on the human prostate cancer cell line PC-3. Oncol Lett 2016; 11 (5): 3179-3184.

18. Coelho SC, Almeida GM, Santos-Silva F, Pereira MC, Coelho MA. Enhancing the efficiency of bortezomib conjugated to pegylated gold nanoparticles: an in vitro study on human pancreatic cancer cells and adenocarcinoma human lung alveolar basal epithelial cells. Expert Opin Drug Deliv 2016; 13 (8): 1075-1081.

19. Guo N, Peng Z, Zhang JJ. Proteasome inhibitor MG132 enhances sensitivity to cisplatin on ovarian carcinoma cells in vitro and in vivo. Int J Gynecol Cancer 2016; 26 (5): 839-844.

20. McBride A, Klaus JO, Stockerl-Goldstein KJ. Carfilzomib: a secondgeneration proteasome inhibitor for the treatment of multiple myeloma. Am J Health Syst Pharm 2015; 72 (5): 353-360.

21. Ruschak AM, Slassi M, Kay LE, Schimmer AD. Novel proteasome inhibitors to overcome bortezomib resistance. J Natl Cancer Inst 2011; 103 (13): 1007-1017.

22. Demo SD, Kirk CJ, Aujay MA et al. Antitumor activity of PR-171, a novel irreversible inhibitor of the proteasome. Cancer Res 2007; 67 (13): 6383-6391.

23. Alsina M, Trudel S, Furman RR et al. A phase I single-agent study of twice-weekly consecutive-day dosing of the proteasome inhibitor carfilzomib in patients with relapsed or refractory multiple myeloma or lymphoma. Clin Cancer Res 2012; 18 (17): 4830-4840.

24. Suzuki E, Demo S, Deu E et al. Molecular mechanisms of bortezomib resistant adenocarcinoma cells. PLoS One 2011; 6 (12): e27996.

25. Luo W, Zhen S, You Y et al. Carfilzomib inhibits cell cycle progression at $\mathrm{G} 2 / \mathrm{M}$ phase and induces apoptosis in breast cancer cells. Int J Clin Exp Med 2016; 9 (11): 22865-22872.

26. Xu Y, Li D, Zeng L et al. Proteasome inhibitor lactacystin enhances cisplatin cytotoxicity by increasing endoplasmic reticulum stress-associated apoptosis in HeLa cells. Mol Med Rep 2015; 11 (1): 189-195.

27. Konac E, Varol N, Kiliccioglu I, Bilen CY. Synergistic effects of cisplatin and proteasome inhibitor bortezomib on human bladder cancer cells. Oncol Lett 2015; 10 (1): 560-564.

28. Kilani S, Sghaier MB, Limem I et al. In vitro evaluation of antibacterial, antioxidant, cytotoxic and apoptotic activities of the tubers infusion and extracts of Cyperus rotundus. Bioresour Technol 2008; 99 (18): 9004-9008.

29. Chou TC, Talalay P. Quantitative analysis of dose-effect relationships: the combined effects of multiple drugs or enzyme inhibitors. Adv Enzyme Regul. 1984; 22: 27-55.

30. Livak KJ, Schmittgen TD. Analysis of relative gene expression data using real-time quantitative PCR and the $2-\Delta \Delta \mathrm{CT}$ method. methods 2001; 25 (4): 402-408.

31. Wagner AD, Grothe W, Haerting J, Kleber G, Grothey A, Fleig WE. Chemotherapy in advanced gastric cancer: a systematic review and metaanalysis based on aggregate data. J Clin Oncol 2006; 24 (18): 2903-2909.

32. Johnston S, Pippen Jr, Pivot X et al. Lapatinib combined with letrozole versus letrozole and placebo as first-line therapy for postmenopausal hormone receptor-positive metastatic breast cancer. J Clin Oncol 2009; 27 (33): 5538-46. 
33. Florea AM, Büsselberg D. Anti-cancer drugs interfere with intracellular calcium signaling. Neurotoxicology 2009; 30 (5): 803-810.

34. Parlati F, Lee SJ, Aujay M et al. Carfilzomib can induce tumor cell death through selective inhibition of the chymotrypsin-like activity of the proteasome. Blood 2009; 114 (16): 3439-3447.

35. O'Connor OA, Stewart AK, Vallone $M$ et al. A phase 1 dose escalation study of the safety and pharmacokinetics of the novel proteasome inhibitor carfilzomib (PR-171) in patients with hematologic malignancies. Clin Cancer Res 2009; 15 (22): 7085-7091.

36. Long Q, Xie Y, Huang $\mathbf{Y}$ et al. Induction of apoptosis and inhibition of angiogenesis by PEGylated liposomal quercetin in both cisplatinsensitive and cisplatin-resistant ovarian cancers. J Biomed Nanotechnol 2013; 9 (6): 965-975.

37. Ao L. Investigating mechanisms determining cancer cell sensitivity to carfilzomib and novel strategies to overcome resistance. University of Kentucky UKnowledge, Theses and Dissertations-Pharmacy, 2016.

38. Baker AF, Hanke NT, Sands BJ et al. Carfilzomib demonstrates broad anti-tumor activity in pre-clinical non-small cell and small cell lung cancer models. J Exp Clin Cancer Res 2014; 33 (1): 111.

39. Konac E, Varol N, Kiliccioglu I, Bilen CY. Synergistic effects of cisplatin and proteasome inhibitor bortezomib on human bladder cancer cells. Oncol Lett 2015; 10 (1): 560-564.
40. Fribley AM, Evenchik B, Zeng Q et al. Proteasome inhibitor PS-341 induces apoptosis in cisplatin-resistant squamous cell carcinoma cells by induction of Noxa. J Biol Chem 2006; 281 (42): 31440-31447.

41. Shen DW, Pouliot LM, Hall MD, Gottesman MM. Cisplatin resistance: a cellular self-defense mechanism resulting from multiple epigenetic and genetic changes. Pharmacol Rev 2012; 64 (3): 706-721.

42. Li XQ, Li J, Shi SB, Chen P, Yu LC, Bao QL. Expression of MRP1, $\mathrm{BCRP}, \mathrm{LRP}$ and ERCC1 as prognostic factors in non-small cell lung cancer patients receiving postoperative cisplatin-based chemotherapy. Int J Biol Marker 2009; 24 (4): 230-237.

43. Farabegoli F, Papi A, Bartolini G, Ostan R, Orlandi M. (-)-Epigallocatechin-3-gallate downregulates Pg-P and BCRP in a tamoxifen resistant MCF-7 cell line. Phytomedicine 2010; 17 (5): 356-362.

44. Dasmahapatra G, Lembersky D, Kramer L et al. The pan-HDAC inhibitor vorinostat potentiates the activity of the proteasome inhibitor carfilzomib in human DLBCL cells in vitro and in vivo. Blood 2010; 115 (22): 4478-4487.

45. Berdeja JG, Hart LL, Mace JR et al. Phase I/II study of the combination of panobinostat and carfilzomib in patients with relapsed/refractory multiple myeloma. Haematologica 2015; 100 (5): 670-676.

Received January 31, 2019. Accepted March 2, 2019. 